\title{
Left atrial voltage remodeling after pulmonary venous isolation with multipolar radiofrequency ablation*
}

\author{
Francesco Laurenzi ${ }^{\#}$, Piergiuseppe De Girolamo, Augusto Pappalardo, Andrea Avella
}

Department of Cardiology, Cardiac Arrhythmia Center, St. Camillo-Forlanini Hospital, Rome, Italy

Email: "

Received 30 August 2013; revised 29 September 2013; accepted 18 October 18, 2013

Copyright (C) 2013 Francesco Laurenzi et al. This is an open access article distributed under the Creative Commons Attribution License, which permits unrestricted use, distribution, and reproduction in any medium, provided the original work is properly cited.

\section{ABSTRACT}

Purpose: Pulmonary vein isolation (PVI) is the accepted primary endpoint for catheter ablation of atrial fibrillation (AF). The aim of this study was to evaluate the level of PVI by PVAC, a multipolar circular catheter utilizing bipolar/unipolar radiofrequency (RF) energy. Methods: Twenty patients with paroxysmal AF underwent PVAC ablation. PVI was validated by voltage reduction and pacing tests. Before and after RF ablation, left atrium (LA) and PV electroanatomic mapping (EAM) were performed by EnSite NavX system. Voltage abatement was considered for potentials $<0.5 \mathrm{mV}$. RF lesion setting was compared to the PVs anatomy previously acquired by a cardiac CT scan. Results: Seventeen patients had four veins and three had a left common PV. All 77 PVs were isolated by PVAC. After RF, EAM showed low voltages areas at the proximal PV ostium and LA. Segmental voltage abatement slightly distal to the anatomic PV ostia was achieved in 20/77 (26\%) PVs, more frequently in veins > 24mm: $9 / 20(45 \%)$ vs $11 / 57$ (19\%), $p<0.05$. Antral lesions were evident in 38/77 PVs (49\%), limited to a part of the antrum in $29 / 38(76 \%)$ veins, with larger occurrence in round than in oval PVs ostia: 25/36 (69\%) vs 13/41 (32\%), p $<$ 0.001. Conclusions: Electrophysiological PVI with PVAC is achieved in all the veins with low voltages areas at the proximal PV ostium. A low voltage circumferential lesion at the anatomic PV ostia is more challenging in larger veins. Antral lesions, frequently affecting part of the antra, were more frequent in round $\mathrm{PV}$ ostia.

Keywords: Atrial Fibrillation; Pulmonary Vein Isolation; Multipolar Circular Ablation Catheter;

${ }^{*}$ Disclosures: None.

"Corresponding author.

\section{Electroanatomic Mapping}

\section{INTRODUCTION}

Different strategies are currently used for atrial fibrillation (AF) catheter ablation, but there is a general consensus on pulmonary vein isolation (PVI) with lesions proximal to the ostia [1]. A debate is still ongoing about the amount of left atrium (LA) substrate proximal to the ostia that should be included in the targeting lesion, as antra can be involved in initiation and maintenance of the arrhythmia. The vast majority of AF catheter ablation procedures rely on point-by-point RF applications and they need a double transseptal puncture and additional techniques such as tree-dimensional (3D) mapping systems or intracardiac echocardiography [1]. To simplify the procedure, different technologies and tools have been designed in order to perform a "one-shot" circular lesion at the PVs ostia. One of these tools is an over-the-wire circular decapolar catheter (PVAC, Medtronic, Minneapolis, MN, USA), capable of mapping and ablating using alternating bipolar-unipolar radiofrequency (RF) energy. The feasibility and efficacy of PVAC-based procedures have been already described with results comparable with point-by-point strategies, shorter procedure time and reduced Rx exposure [2-8]. The aim of this study was to analyse the PVAC lesion setting through a 3D impedance-based electroanatomic mapping (EAM), to assess how proximal the lesion extension is with respect to the PV-LA junction and how it is influenced by anatomical variants.

\section{METHODS}

\subsection{Patients}

The study group included patients with symptomatic paroxysmal AF refractory to antiarrhythmic drugs, who underwent bipolar-unipolar RF ablation and EAM, be- 
tween January and November 2012. Exclusion criteria were the presence of a large left atrium (long axis diameter $>50 \mathrm{~mm}$ ), left appendage thrombus, left ventricular ejection fraction (LVEF) $<45 \%$, severe mitral valve disease, severe renal or pulmonary comorbidity, contraindication to oral coagulation and pregnancy.

\subsection{Electrophysiological Study and 3D Voltage Mapping}

All patients received oral anticoagulation therapy for at least 4 weeks up to 3 days before the procedure, then replaced by a low molecular weight heparin. A computed tomography (CT) scan of the heart and a transoesophageal echocardiogram were performed within 48 hours before the procedure. Surface and bipolar electrocardiograms (ECGs) were continuously monitored and recorded. LA access was gained through a single transseptal puncture to position a steerable $9.5 \mathrm{~F}$ sheath (Channel, Bard EP, Lowell, MA, USA), continuously perfused with $0.9 \%$ saline solution. After the transseptal puncture, intravenous heparin was administered as a bolus (100 $\mathrm{IU} / \mathrm{kg}$ ) followed by infusion, to achieve and maintain a target activated clotting time of $350 \mathrm{~s}$.

A $3 \mathrm{D}$ reconstruction of the LA and PVs was performed using the NavX system (EnSite NavX, St Jude Medical, St Paul, MN, USA). A circular multipolar mapping catheter (Inquiry A-Focus II, St Jude Medical) was used for the acquisition of the 3D geometry, which was then compared side by side to the CT scan-derived anatomy. PVs ostia were tagged at the intersection of the tangential lines to PVs and LA walls [9]. A common PV was defined as a unique take-off of contiguous PVs, bifurcating $>5 \mathrm{~mm}$ distal to the PV-LA junction. The proximal border of the antra has not been standardized yet, but arbitrarily we judged the antral extension for lesions occurring $>5 \mathrm{~mm}$ from the PVs ostia. PVs ostia were anatomically characterized by measurement of the maximum and minimum diameter and the relative ratio, defining round shaped ostia for a value $\leq 1.2$ and oval shaped ostia for a ratio $>1.2$ [10]. Bipolar voltage maps were acquired before and after PVs isolation in sinus rhythm. The following settings were used: interpolation of $10 \mathrm{~mm}$, internal and external projections of $5 \mathrm{~mm}$. Normal ECGs were evaluated for voltage recordings greater than $0.5 \mathrm{mV}$ and were displayed in purple. Voltage abatement was considered for potentials ranging from 0.5 to $0.05 \mathrm{mV}$ displayed in a spectrum of colours ranging from red to blue, and for signals lower than 0.05 $\mathrm{mV}$ marked in grey and conventionally considered as electrically silent [11]. Off-line quantitative voltage analysis was performed collecting voltage values of points mapped at each PV-LA junction and relative proximal segment of the vein, listed as below: LSPV for the left superior PV, LIPV for the left inferior PV, LCPV for the left common PV, RSPV for the right superior PV and RIPV for the right inferior PV.

\subsection{Multipolar Radiofrequency Catheter Ablation}

Ablation of PVs was performed using the PVAC, a decapolar circular over-the-wire catheter capable of mapping and ablation. The distal circular loop, measuring 25 $\mathrm{mm}$ in diameter, can be steered in a bidirectional fashion and extended in a spiral configuration. The ablation requires a specifically designed RF generator (GENius, Ablation Frontiers, Medtronic, USA), that allows RF delivery in various settings of bipolar/unipolar energy output and, through continuous temperature monitoring in each electrode, restrict the power output to a maximum of 10 watts. The location of the decapolar ring was checked both using fluoroscopy and the 3D PVs-LA reconstruction. RF energy was delivered for $60 \mathrm{~s}$ with a temperature limit of $60^{\circ} \mathrm{C}$, with a bipolar/uniratio of $2: 1$ when the catheter was in close proximity to the PV antrum and 4:1 if the position was more ostial. Cavotricuspid isthmus ablation was also performed using an 8-mm tip RF ablation catheter if a typical right atrial flutter was observed during the procedure or previously documented.

\subsection{Confirmation of Pulmonary Vein Isolation}

Before NavX remapping, PVI was always checked using the PVAC and then a standard circular multipolar catheter (Inquiry A-Focus II, St Jude Medical). Electrical deconnection was assessed by abatement of local potentials in sinus rhythm and during atrial pacing and by achievement of PVs-LA conduction block. Entry conduction block was demonstrated by the absence or dissociation of PVs potentials. Exit block was assessed by pacing at high output ( $10 \mathrm{~V}$ at $2 \mathrm{~ms}$ ) from all electrode pairs of the catheter, positioned in the proximal PVs and at the ostia. Persistence of PVs isolation was assessed again in all the PVs at the end of the study. No pharmacological tests were performed.

\subsection{Statistical Analysis}

Statistical analysis was performed using SPSS 12.0 for Windows (SPSS, Inc., Chicago, IL, USA) Continuous data are expressed as mean values \pm standard deviation. Categorical variables are expressed as a percentage. Differences between distributions were compared with a paired t-test for paired data and unpaired t-test for independent values. Differences in proportions were compared with a Fisher's exact test. A P value $<0.05$ was considered statistically significant. 


\section{RESULTS}

\subsection{Patient Characteristics}

PVAC lesion setting was evaluated in twenty patients who underwent alternating bipolar/unipolar RF ablation and impedance-based EAM. Mean age of the patients was $59 \pm 10$ years, 14 were males and 6 females. All the patients suffered from drug-refractory paroxysmal atrial fibrillation with a mean CHADS2 Score of $1.2 \pm 0.4$. Echocardiographic recordings demonstrated a mean LA diameter of $41 \pm 5 \mathrm{~mm}$ and a mean LVEF of $55 \% \pm 6 \%$.

PVs anatomy, ostial size, and shape are summarized in Table 1. Seventeen patients had 4 veins each (LSPV, LIPV, RSPV and RIPV) and three patients had three veins (left common PV, RSPV and RIPV). The PV ostial shape was classified as oval in 41/77 (53\%) and round in $36 / 77$ (47\%) veins. Oval ostia had a greater incidence in left than in right PVs: $24 / 37$ (65\%) vs 17/40 (43\%), p = ns. Round ostia were more frequently in right than in left PVs: $23 / 40(57 \%)$ vs $13 / 37$ (35\%), $p=n s$.

\subsection{Multipolar Radiofrequency Catheter Ablation}

PVAC was able to target and isolate all the $77 \mathrm{PVs}$, including the three LCPVs, independently from size and shape of the PV ostium. The mean number of duty-cycled RF applications per patient was $26 \pm 9$ (range 19 - 44) with a mean energy delivery time of $24 \pm 6$ (range 19 40) min. The fluoroscopy time was $51 \pm 14$ min, including both the time for PVAC mapping and ablation and the time due to catheter manipulation for voltage mapping. Five patients also underwent cavotricuspid isthmus ablation.

\subsection{D Voltage Mapping}

The voltage maps were collected before and after PVI through the acquisition of $343 \pm 63$ and $371 \pm 85$ points, respectively $(\mathrm{p}=\mathrm{ns})$. Before ablation all the points mapped at the PVs antra and in the proximal tubular portion of the veins had a normal voltage (Figures 1(a) and (b)). After ablation a voltage reduction was evident in all the PVs and to different extent proximal to their ostia (Figures 1(c) and (d)), with maps coloured grey (voltage $<0.05 \mathrm{mV}$ ) and red-blue (voltage between 0.05 and 0.5 $\mathrm{mV})$. A quantitative voltage analysis showed a significant voltage reduction in all the veins and proximal PVs ostia (Figure 2): $84 \%$ for the LSPV, $88 \%$ for the LIPV, $86 \%$ for the LCPV, $87 \%$ for the RSPV and $81 \%$ for the RIPV $(\mathrm{p}<0.05)$. Mean voltages recorded in the individual PVs were not significantly different before or after RF ablation.

The qualitative voltage map analysis of RF lesion setting in respect of ostial shape for each PV is shown in
Table 1. PVs ostia: size and shape.

\begin{tabular}{cccccc}
\hline & $\begin{array}{c}\text { Maximum } \\
\text { diameter } \\
(\mathbf{m m})\end{array}$ & $\begin{array}{c}\text { Minimum } \\
\text { diameter } \\
(\mathbf{m m})\end{array}$ & $\begin{array}{c}\text { Diameter } \\
\text { ratio }\end{array}$ & $\begin{array}{c}\text { Round } \\
\text { PV ostia PV ostia } \\
\text { (n) }\end{array}$ & $\begin{array}{c}\text { Oval } \\
\text { (n) }\end{array}$ \\
\hline LSPV & $21.2 \pm 4.2$ & $17.0 \pm 3.8$ & $1.28 \pm 0.3$ & 7 & 10 \\
LIPV & $19.1 \pm 2.7$ & $15.0 \pm 2.8$ & $1.26 \pm 0.2$ & 6 & 11 \\
$\mathbf{L C P V}$ & $30.6 \pm 1.5$ & $22.7 \pm 1.1$ & $1.35 \pm 0.0$ & 0 & 3 \\
$\mathbf{R S P V}$ & $20.2 \pm 5.0$ & $18.8 \pm 4.7$ & $1.14 \pm 0.1$ & 12 & 8 \\
RIPV & $18.7 \pm 3.9$ & $16.8 \pm 3.1$ & $1.19 \pm 0.2$ & 11 & 9 \\
\hline
\end{tabular}

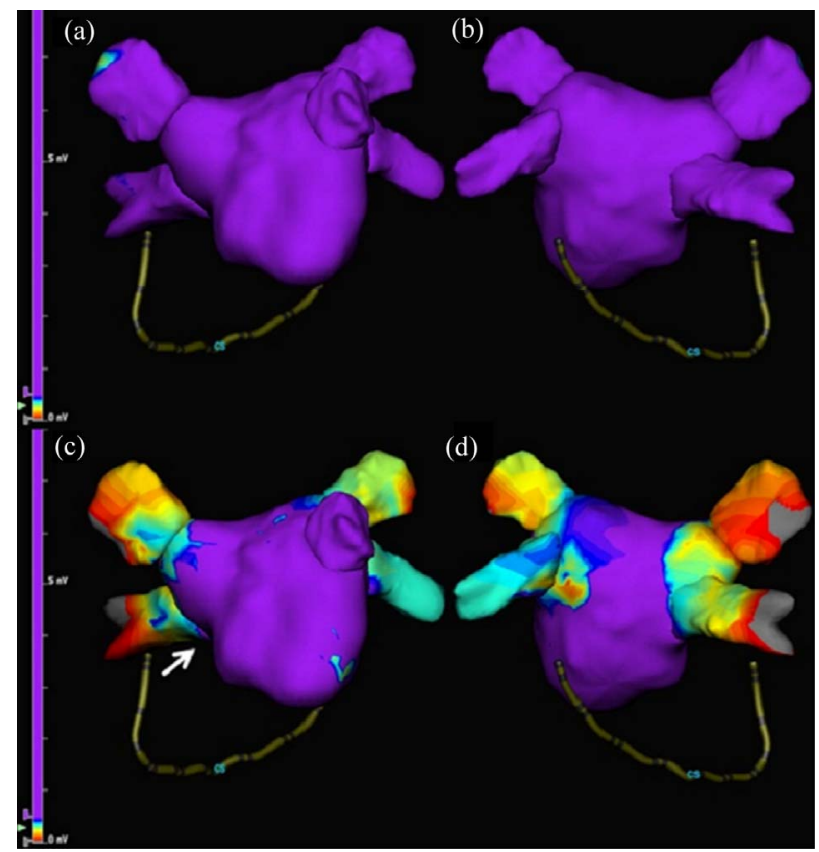

Figure 1. Voltage maps before and after pulmonary vein isolation. Anterior and posterior views of LA voltage maps, before ((a) and (b)) and after ((c) and (d)) PVI by PVAC. Purple colour identifies areas of normal voltage $(>0.5 \mathrm{mV})$, colour ranging from red to blue areas with low voltages $(<0.5 \mathrm{mV})$ and gray colour areas of very low voltage $(<0.05 \mathrm{mV})$. After PVI, voltage abatement was evident at PV ostia and proximal LA. The arrow indicates a small area of voltage abatement just distal to the anatomic right inferior PV ostia.

Figure 3. A reduced segmental voltage abatement was observed at the anatomic PV ostia in 20/77 (26\%) PVs, with a full low voltage circumferential area slightly distal to the ostia. This event was more frequent in veins with a maximum diameter larger than $24 \mathrm{~mm}$ : $9 / 20(45 \%)$ vs $11 / 57$ (19\%), $\mathrm{p}<0.05$, (Table 2). No difference was observed in between left vs right PVs, $12 / 37(32 \%)$ vs $8 / 40$ $(20 \%) \mathrm{p}=\mathrm{ns}$, and round vs oval PV ostia, 11/36 (30\%) vs $9 / 41(22 \%) \mathrm{p}=\mathrm{ns}$.

Ostial RF lesions were detected in 39/77 (51\%) veins and antral lesions in 38/77 PVs (49\%). Ostial lesions were more frequent in oval than in round PV ostia, 28/41 $(68 \%)$ vs $11 / 36(31 \%)$, without any difference in left vs right PVs, $23 / 37(62 \%)$ vs $16 / 40(40 \%) \mathrm{p}=\mathrm{ns}$, nor in 


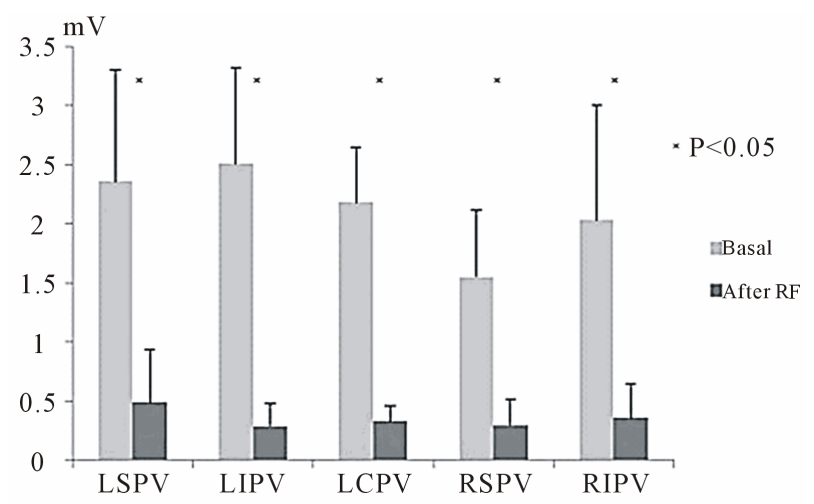

Figure 2. Voltage before and after pulmonary vein isolation. Mean voltage values $(\mathrm{mV})$ before (light bars) and after (dark bars) PVI. LSPV: left superior PV; LIPV: left inferior PV; LCPV: left common PV; RSPV: right superior PV; RIPV: right inferior PV.
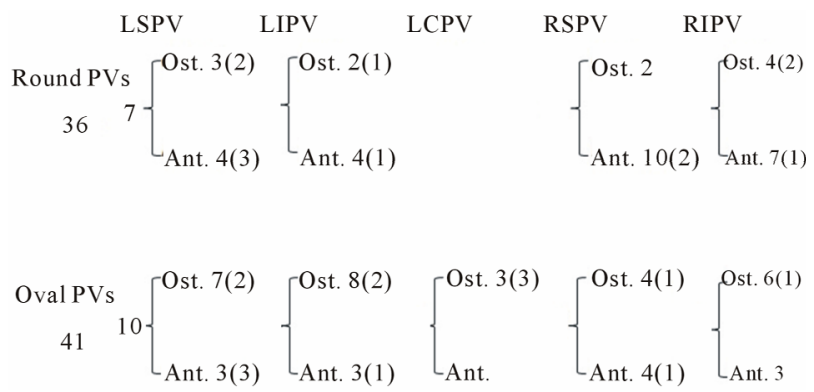

Figure 3. Lesion setting and ostial shape in each PV. Abbreviations as in Table 1. In brackets are indicated the veins with a segmental voltage abatement slightly distal to the anatomic PV ostia.

veins with a diameter larger than $24 \mathrm{~mm}, 23 / 37(62 \%)$ vs $16 / 40(40 \%), p=n s$.

Antral lesions had a greater occurrence in round than in oval PV ostia: 25/36 (69\%) vs 13/41(32\%), p $<0.001$. No any difference was observed in left vs right PVs, $14 / 37(38 \%)$ vs $24 / 40(60 \%) \mathrm{p}=\mathrm{ns}$, nor in veins larger than $24 \mathrm{~mm}, 8 / 20(40 \%)$ vs $30 / 57(53 \%)$, $\mathrm{p}=$ ns.

Antral extension of RF lesions were evident in the whole antra in 9/38 veins and limited to a part of the PV antrum in 29/38 (76\%) PVs: the posterior wall in 24 and the anterior wall in $5 \mathrm{PV}$ antra.

\section{DISCUSSION}

Our data confirm the results of a previous study [12] about the ability of PVAC to perform PVI at the proximal ostium, even in more challenging anatomical variants as three left common PVs, in which ostial lesions were carried out by insertion of the guide in different venous branches.

After RF ablation, the impedance-based EAM showed a significant reduction in the bipolar voltage at the PV ostia, proximal to the tubular part of the PVs. However about one in four veins, frequently the larger ones, showed a segment of reduced voltage abatement at the anatomic PV ostium with slightly distal low voltage encircling. Far-field signal recording, particularly frequent in left-sided veins because of their proximity to the left atrial appendage, was ruled out by remapping with standard catheters and pacing maneuvers. On the other hand, a partial sided placement of the PVAC at the ostia may be the cause of the distal segmental voltage abatement: before $\mathrm{RF}$ erogation in any new catheter position, the location of the PVAC was displayed on the 3D mapping system, but a tiny displacement may have occurred as a consequence of the pressure exerted during RF delivery in order to achieve a better contact and the target temperature.

In our experience antral extension of RF lesions was achieved more frequently in round veins, as counterclockwise catheter rotation allows a wider sweep more easily around the circumference of round PV ostia. More often the antra were partially affected by RF lesions, probably as a result of different causes. Firstly, the PV's short axis is oriented approximately in the anteroposterior direction. Secondly, the transseptal access naturally directs the catheter posteriorly into the LA, so that its circular ending is obliquely oriented with the anterior part located at the PV ostia and the posterior part more atrial. Finally, a greater antral contact was more easily achieved posteriorly by bending and rotating the catheter around the ostial circumference. On the contrary, the small distance between the left PVs and the LA appendage [10] can limit the placement of the circular catheter in the anterior LA walls more than that of a single-tip catheter.

In our study the fluoroscopic and procedural mean times were longer than those previously described [2-8], because the procedure required an additional EAM and probably a different learning curve, since the mean number of RF applications was similar.

The PVAC is an over-the-wire catheter designed to be managed under fluoroscopic control, with more detailed definition of the PV-LA junction usually achieved by angiography. A small risk of PV stenosis is described in some studies $[13,14]$. In our experience the 3D-geometry reconstruction eased the positioning of the PVAC at the ostia and the parallel orientation to the PV's walls, but was not useful for catheter navigation as it is conditioned by the over-the-wire design. Regarding the voltage maping, the low voltage signals recorded are not accurate enough to indicate PVI, which in fact was validated with electrophysiological entry and exit blocks. Moreover, maps can be affected by far-field potentials, particularly from the left atrial appendage, thus requiring accurate characteristics analysis and pacing maneuvers. As the PVAC-EAM combined strategy is more expensive and 
Table 2. Qualitative voltage map analysis.

\begin{tabular}{|c|c|c|}
\hline \multicolumn{2}{|c|}{ Segmental low voltage area distal to the anatomic PV ostia n. 20/77 } & \multirow{2}{*}{$\begin{array}{c}\text { p value } \\
0.30\end{array}$} \\
\hline Left vs right PVs & $12 / 37(32 \%)$ vs $8 / 40(20 \%)$ & \\
\hline Round vs oval PV ostia & $11 / 36(30 \%)$ vs $9 / 41(22 \%)$ & 0.44 \\
\hline PV diameter $>24$ vs $<24 \mathrm{~mm}$ & $9 / 20(45 \%)$ vs $11 / 57(19 \%)$ & 0.037 \\
\hline \multicolumn{3}{|c|}{ Ostial lesions n. 39/77 } \\
\hline Left vs right PVs & $23 / 37(62 \%)$ vs $16 / 40(40 \%)$ & 0.069 \\
\hline Round vs oval PV ostia & $11 / 36(31 \%)$ vs $28 / 41(68 \%)$ & 0.001 \\
\hline PV diameter $>24$ vs $<24 \mathrm{~mm}$ & $12 / 20(60 \%)$ vs $27 / 57(47 \%)$ & 0.44 \\
\hline \multicolumn{3}{|c|}{ Antral lesion n. 38/77 } \\
\hline Left vs right PVs & $14 / 37(38 \%)$ vs $24 / 40(60 \%)$ & 0.069 \\
\hline Round vs oval PV ostia & $25 / 36(69 \%)$ vs $13 / 41(32 \%)$ & 0.001 \\
\hline PV diameter $>24$ vs $<24 \mathrm{~mm}$ & $8 / 20(40 \%)$ vs $30 / 57(53 \%)$ & 0.44 \\
\hline
\end{tabular}

time consuming, other techniques such as angiography or 3D CT/MRI cardiac scan reconstruction are to be preferred for PV-LA junction definition.

This study outlines the significance of two factors in order to choose the more effective ablation tool: the knowledge of the individual anatomy that is useful to perform a right positioning of the circular mapping-ablation catheter, and the a priori preference of a wide encircling PVI strategy that is easier to achieve with a single-point RF catheter.

\section{Limitations}

This study analyzed the primary lesion setting after PVAC ablation in a relatively small but representative group of patients, referred for paroxysmal AF catheter ablation.

PVI was assessed by standard electrophysiological criteria as there is no absolute agreement between myocardial viability and low voltages and also because the maps can be affected by LA far-field potentials. As repeated mapping procedures were not performed, the study does not clarify to what extent the ablation lesions remain permanent.

\section{CONCLUSION}

Electrical PVI with PVAC is not affected by the size and shape of the PV ostium and results in low voltage areas mainly at the PV ostium and proximal LA. However, voltage reduction in the whole circumference of the anatomic PV ostia is more challenging in larger veins, and antral lesions are variable in occurrence and extension, so knowledge of the individual anatomy of the PVs and LA can be useful in order to choose the more effective ablation tool.

\section{ACKNOWLEDGEMENTS}

The authors wish to thank Simona Benedetti, Roberta Annibali and Sergio Orsini for technical assistance and data collection and Tiziana
De Santo for statistics.

\section{REFERENCES}

[1] Calkins, H., Kuck, K.H., Cappato, R., Brugada, J., Camm, J., Chen, S.A., Crijns, H.J., Damiano, R.J., Davies, W., Di Marco, J., Edgerton, J., Ellenbogen, K., Ezekowitz, M.D., Haines, D.E., Haissaguerre, M., Hindricks, G., Iesaka, Y., Jackman, W., Jalife, J., Jais, P., Kalman, J., Keane, D., Kim, Y.H., Kirchhof, P., Klein, G., Kottkamp, H., Kumagai, K., Lindsay, B.D., Mansour, M., Marchlinski, F.E., McCarthy, P.M., Mont, L., Morady, F., Nademanee, K., Nakagawa, H., Natale, A., Nattel, S., Packer, D.L., Pappone, C., Prystowsky, E., Raviele, A., Reddy, V., Ruskin, J.N., Shemin, R.J., Tsao, H.M. and Wilber, D. (2012) HRS/EHRA/ECAS Expert consensus statement on catheter and surgical ablation of atrial fibrillation: Recommendations for patient selection, procedural techniques, patient management and follow-up, definitions, endpoints, and research trial design. Heart Rhythm, 9, 632-696.

[2] Boersma, L., Wijffels, M., Oral, H., Wever, E. and Morady, F. (2008) Pulmonary vein isolation by duty-cycled bipolar and unipolar radiofrequency energy with a multielectrode ablation catheter. Heart Rhythm, 5, 16351642.

[3] Fredersdorf, S., Weber, S., Jilek, C., Heinicke, N., Von Bary, C., Jungbauer, C., Riegger, G.A., Hamer, O. and Jeron, A. (2009) Safe and rapid isolation of pulmonary veins using a novel circular ablation catheter and dutycycled RF generator. Journal of Cardiovascular Electrophysiology, 20, 1097-1101. http://dx.doi.org/10.1111/j.1540-8167.2009.01501.x

[4] Wiekzorek, M., Hoeltgen, R., Akin, E., Salili, A.R., Oral, H. and Morady, F. (2010) Results of short-term and long-term pulmonary vein isolation for paroxysmal atrial fibrillation using duty-cycled bipolar and unipolar radiofrequency energy. Journal of Cardiovascular Electrophysiology, 21, 399-405.

http://dx.doi.org/10.1111/j.1540-8167.2009.01640.x

[5] Duytschaever, M., Anne, W., Papiashvili, G., Vandekerckhove, Y. and Tavernier, R. (2010) Mapping and isolation of the pulmonary veins using the PVAC catheter. 
Pacing and Clinical Electrophysiology, 33, 168-178. http://dx.doi.org/10.1111/j.1540-8159.2009.02609.x

[6] Bulava, A., Hanis, J., Sitek, D., Osmera, O., Karpianus, D., Snorek, M., Rehouskova, K., Tousek, F. and Pesl, L. (2010) Catheter ablation for paroxysmal atrial fibrillation: A randomized comparison between multielectrode catheter and point-by-point ablation. Pacing and Clinical Electrophysiology, 33, 1039-1046. http://dx.doi.org/10.1111/j.1540-8159.2010.02807.x

[7] Bittner, A., Mönnig, G., Zellerhoff, S., Pott, C., Köbe, J., Dechering, D., Millberg, P., Wasmer, K. and Eckardt, L. (2011) Randomized study comparing duty-cycled bipolar and unipolar radiofrequency with point by point ablation in pulmonary vein isolation. Heart Rhythm, 8, 1383-1390. http://dx.doi.org/10.1016/j.hrthm.2011.03.051

[8] Mulder, A.A., Wijffels, M.C., Wever, E.F. and Boersma, L.V. (2012) Freedom from paroxysmal atrial fibrillation after successful pulmonary vein isolation with pulmonary vein ablation catheter-phased radiofrequency energy: 2year follow-up and predictors of failure. EP Europace, 14, 818-825. http://dx.doi.org/10.1093/europace/eus010

[9] Ho, S.Y., Cabrera, J.A., Tran, V.H., Farré, J., Anderson, R.H. and Sánchez-Quintana, D. (2001) Architecture of the pulmonary veins: relevance to radiofrequency ablation. Heart, 86, 265-270. http://dx.doi.org/10.1136/heart.86.3.265

[10] Schmidt, B., Ernst, S., Ouyang, F., Chun, J., Broemel, T., Bänsch, D., Kuck, K.H. and Antz, M. (2006) External and endoluminal analysis of left atrial anatomy and the pulmonary veins in three-dimensional reconstructions of magnetic resonance angiography: The full insight from inside. Journal of Cardiovascular Electrophysiology, 17, 957-964. http://dx.doi.org/10.1111/j.1540-8167.2006.00548.x

[11] Chang, S.L., Tai, C.T., Lin, Y.J., Wongcharoen, W., Lo, L.W., Tuan, T.C, Udyavar A.R., Chang, S.H., Tsao, H.M., Hsieh, M.H., Hu, Y.F., Chen, Y.J. and Chen, A.S. (2007) Biatrial substrate properties in patients with atrial fibrillation. Journal of Cardiovascular Electrophysiology, 18, 1134-1139. http://dx.doi.org/10.1111/j.1540-8167.2007.00941.x

[12] Raffa, S., Große, A., Brunelli, M., Wauters, K. and Geller, J.C. (2010) Voltage mapping and pacing to assess the level of pulmonary isolation achieved with a novel circular multielectrode ablation catheter. EP Europace, 12, 933-940.

[13] Von Bary, C., Weber, S., Dornia, C., Eissnert, C., Fellner, C., Latzin, P., Fredersdorf, S., Stadler, S. and Hamer, O.W. (2011) Evaluation of pulmonary vein stenosis after pulmonary vein isolation using a novel circular mapping and ablation catheter (PVAC). Circulation: Arrhythmia and Electrophysiology, 4, 630-636. http://dx.doi.org/10.1161/CIRCEP.111.963397

[14] De Greef, Y., Tavernier, R., Raeymaeckers, S., Schwagten, B., Desurgeloose, D., De Keulenaer, G., Stockman, D., De Buyzere, M. and Duytschaever, M. (2012) Prevalence, characteristics and predictors of pulmonary vein narrowing after PVAC ablation. Circulation: Arrhythmia and Electrophysiology, 5, 52-60. http://dx.doi.org/10.1161/CIRCEP.111.961888 